\title{
AN INSTRUMENT FOR LUNAR SURFACE CHEMICAL ANALYSIS
}

\section{Anthony Turkevich, Karlfried Knolle}

Enrico Fermi Institute for Nuclear Studies

$$
\text { and. }
$$

Department of Chemistry

University of Chicago, Chicago, Illinois

Richard E. Emmert, Wayne A. Anderson

Laboratory for Astrophysics and Space Research of the Enrico Fermi Institute for Nuclear Studies University of Chicago, Chicago, Illinois

James H. Patterson

Chemistry Division

Argonne National Laboratory, Argonne, Illinois

and.

Ernest Franzgrote

Jet Propulsion Laboratory, Pasadena, California

Submitted to

The Review of Scientific Instruments

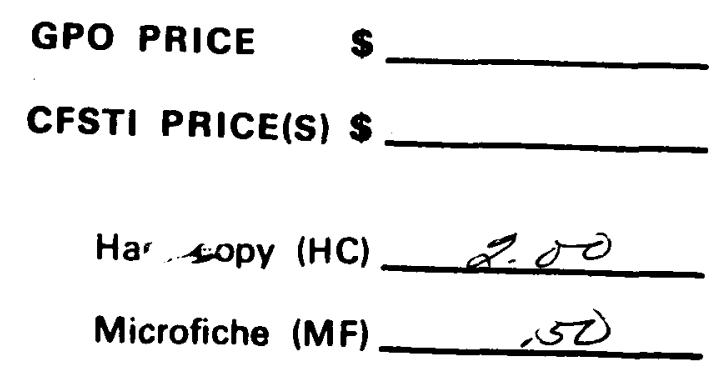

May 1966
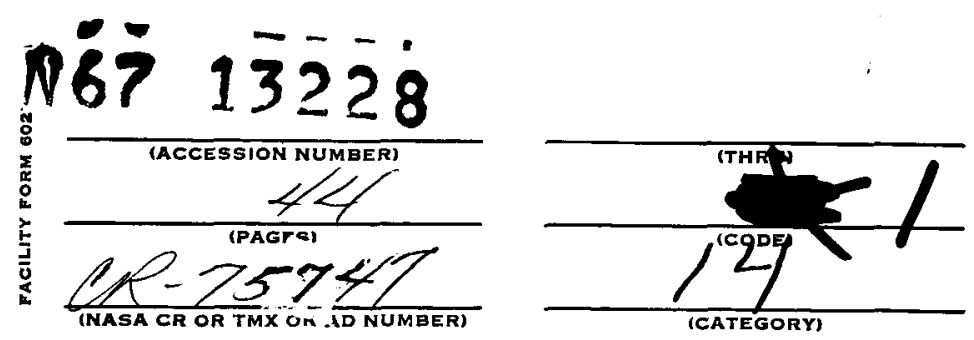
An Instrument for Lunar Surface Chemical Analysis*

Anthony Turkevich, Karlfried Knolle ${ }^{\dagger}$

Enrico Fermi Institute for Nuclear Studies

and.

Department of Chemistry

University of Chicago, Chicago, Illinois

Richard E. Emmert, ${ }^{\ddagger}$ wayne A. Anderson

Laboratory for Astrophysics and Space Research

of the Enrico Fermi Institute for Nuclear Studies

University of Chicago, Chicago, Illinois

James H. Patterson

Chemistry Division

Argonne National Laboratory, Argonne, Illinois

and.

Ernest Franzgrote

Jet Propulsion Laboratory, Pasadena, California

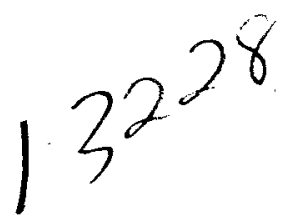

An instrument has been designed and constructed which utilizes the interactions with matter of monoenergetic alpha particles for surface chemical analysis. The source of alpha particles is a radioactive nuclide, such as $\mathrm{cm}^{242}$. The energy spectra of backscattered. alpha particles are measured with semiconductor detectors. Other detectors (with gold foils over them to eliminate alpha particles) register protons from $(\alpha, p)$ reactions. Transistorized electronics provide 128channel pulse-height analyzers for both alpha and proton spectra. The specific unit designed for the Surveyor Lunar Mission weighs less than $4 \mathrm{~kg}$. and uses less than 1.3 watts. 


\section{INTRODUCTION}

The interactions with matter of alpha particles from radioactive sources can be made the basis of an analytical technique. The principles of this method have been described elsewhere. ${ }^{1,2}$ It takes advantage of the characteristic energy spectra of alpha particles elastically scattered backward and the spectra of protons produced by $(\alpha, p)$ reactions, to deduce the chemical composition of the sample being exposed to the alpha particles. A convenient source of monoenergetic alpha particles for such a purpose is an alpha radioactive nuclide, such as $\mathrm{Cm}^{242}$. The energy spectra of the alpha particles and. protons are efficiently determined, using semiconductor detectors and transistorized electronics. The present report describes a prototype instrument constructed according to these principles and designed for the chemical analyses of lunar and. planetary surfaces. The electronics of this instrument have been adapted from those employed in the University of Chicago cosmic-ray programs.

Because of requirements peculiar to the proposed lunar mission, the system is divided into two functional blocks: a deployable sensor head ("Head. Unit") and a digital electronics system ("Electronics Unit"). The sensor head is designed to be placed directly on the lunar surface. It contains the alpha radioactive sources, semiconductor detectors, and the linear portion of the electronics (through height-to-time signal conversion). The digital electronics unit contains a gated clock 
for pulse width to digital conversion, temporary data storage, command switching, power distribution, and spacecraft interface circuitry. Figure 1 shows pictures of the two parts of the first prototype instrument which is described in this report, together with the test rack used for checking its behavior. The total weight of the two components of this unit is $3.5 \mathrm{~kg}$, with $1.4 \mathrm{~kg}$. of this being the weight of the deployable head. The power consumption of the instrument is less than 1.3 watts most of it in the electronics package. It is designed to operate from a 24-volt supply. Details of the sub-systems, as well as data on the performance, are provided in the following sections.

\section{II. "HEAD" UNIT}

\section{A. General Characteristics}

The "Head" Unit of the instrument (see Fig. 1) is a box $13.3 \mathrm{~cm}$ by $17.1 \mathrm{~cm}$ by $11.7 \mathrm{~cm}$ high, containing the alpha sources, the detectors, and part of the transistorized electronics. It has a circular opening in the bottom $10.8 \mathrm{~cm}$ in diameter. The sample is examined through this opening. Figure 2 shows a view, through the bottom opening, of the head unit. Figure 3 shows some details of construction. In a lunar operation, the head unit would be deployed on to the original (or on to a prepared) surface of the moon. In laboratory studies, the head unit is kept in a vacuum and samples are presented to it through a vacuum lock. 
The components of the head are designed to allow the sample to be uniformly irradiated with a collimated stream of alpha particles from the source holders. Alpha particles scattered backwards from the sample are detected by the two energy sensitive semiconductor detectors (alpha detectors). Protons produced by the alpha particles in the sample are detected by the four symmetrically placed proton detectors.

\section{B. Sources}

The alpha particles come from six collimated source holders. The source holders are separate units that are designed. to be inserted from the top of the head turret for ease of replacement and to minimize the danger of contamination of the part of the instrument that can be seen by the detectors. The details of the construction are shown in Fig. 4. The collimators are made of stainless steel. The dimensions in these collimators are very critical, since they determine the direction of the alpha particles so that they strike only the sample aperture $5.7 \mathrm{~cm}$ away. Likewise, very important are pumping passageways which allow the air to be pumped out from the space between the sources and the collimator faces. This air is conducted to the top of the turret head, again to minimize the danger of contamination of the sensitive parts of the instrument.

Across the face of each collimator is placed a thin film to prevent recoils from the alpha source from reaching the sample area or the sensitive part of the instrument. This film must be thick enough to stop the recoiling atoms but not thick 
enough to degrade the energy or homogeneity of the source. Aluminum oxide films of approximately $1000 \AA$ thickness ${ }^{*}$ have been found to be stable to the radiation from $10^{10} \mathrm{~d} / \mathrm{m}$ of $\mathrm{Cm}^{242}$ for a period of six months. Organic films appear to be much more susceptible to radiation damage. The collimator, together with the protective film, is a replaceable section of the source holder (see Fig. 4).

The ideal isotope for the sources would emit alpha particles with a single energy that would be greater than $5.8 \mathrm{MeV}$. It should have no gamma activity. Its half life would be about two years and that of its first daughter at least $10^{3}$ years. It should be available commercially in curie quantities, free of non-volatile and radioactive impurities. Chemically, it should. be readily purified from all other non-volatile elements and give stable, adherent deposits on plates at alpha-activity levels up to 0.5 curie $/ \mathrm{cm}^{2}$.

A brief trial of $\mathrm{PO}^{210}$, which is commercially available and practically monoenergetic, with a non-radioactive daughter, was abandoned because contamination problems were difficult to control. $\mathrm{Cm}^{242}$ was found to fit the requirements as closely as any available isotope. Its main peak has an energy of $6.11 \mathrm{MeV}$. The only other one of significance is $44 \mathrm{KeV}$ lower in energy, with about one third its intensity. Although the ratio of gamma to alpha events is only about $4 \cdot 10^{-4}$ because of internal conversion, there is enough low-energy gamma emission associated with the high level of alpha activity to require consideration as a * Procured from the Rauland Corp., Chicago, Illinois. 
source background in the lower energy channels of the present system. Likewise, a small amount of spontaneous fission gives rise to a few neutrons. The lower than optimum half life (162 days) makes it necessary to correct for decay of the sources for experiments lasting longer than a few days. This also limits the useful life of a source because of its decrease in strength, and because of the growth of its daughter $\mathrm{Pu}^{238}$, with a half life of 89 years.

$\mathrm{Cm}^{242}$ is not commercially available at this time but may be prepared in curie quantities by irradiation of $\mathrm{Am}^{241}$ in a nuclear reactor, followed by chemical separation of the curium. 3 The concentrations of the longer lived isotopic impurities, $\mathrm{Cm}^{243}$ and $\mathrm{Cm}^{244}$ (half lives 32 years and 18 years, respectively), are the principal limitation to the useful life of curium from a given irradiation, as their activity becomes relatively more important with the decay of the $\mathrm{Cm}^{242}$. Their presence is kept as low as possible by irradiating a large amount of $\mathrm{Am}^{241}$, free of $\mathrm{Am}^{243}$, with the minimum number of neutrons necessary to obtain the desired quantity of $\mathrm{cm}^{242}$. Chemically, curium can be manipulated. without difficulty, and the sources obtained are adherent enough so that no losses have been observed through shaking, air currents, evaporation, or creeping. However, activity can be rubbed. off readily. These sources originally have energy spectra with full widths at half maximum of $0.5-2.0 \%$. However, unless the sources are protected from atmospheric humidity, they deteriorate, with the widths reaching $~ 3 \%$ in a week, and low-energy tails appearing in the spectra. 
The sources for the present instrument were prepared by evaporating the chemically purified curium onto stainless steel plates which were then inserted into the source holders. The usable area of the sources is $0.066 \mathrm{~cm}^{2}$. About 2.2 per cent of the alpha particles get out through the collimating system. The original source strength of the individual six sources used in most of the work with this instrument was between 1 and $3 \cdot 10^{10} \mathrm{~d} / \mathrm{m}$. If larger source strengths than the ones employed here are to be used, it might be necessary to reduce interference by constructing the turret in the vicinity of the sources out of a heavy material, such as Hevi-met, (a tungsten alloy).

C. The Scattered Alpha Mode of the Instrument

This mode of the instrument is designed to detect and determine the energy of alpha particles scattered by the sample. Although the energy of such scattered alpha particles is not a sensitive function of the angle in the backward direction, ${ }^{2}$ the distances from the source to the sample and the sample to the alpha detector were made relatively large $(5.7 \mathrm{~cm})$, at the cost of increased measurement time, to minimize possible effects of surface roughness. ${ }^{4}$ At a scattering angle of $180^{\circ}$, all such effects should rigorously disappear. In the present instrument, the average scattering angle was $174.5^{\circ}$, with a range of one degree on each side of this value.

The two alpha detectors are of the silicon surface barrier type. Details of construction are shown in Fig. 4. Masks in front of the detectors, with an opening of $0.20 \mathrm{~cm}^{2}$, prevent 
alpha particles from reaching the edge areas of non-uniform sensitivity, and also keep the detectors from seeing the tips of the source collimators which might be contaminated.

The detectors are reverse-biased to produce an active depth $(\sim 50 \mu)$, deep enough to generate full energy pulses from 7-MeV alpha particles, but not deep enough for significant energy deposition by protons or electrons, either from the sample or from cosmic rays. Such detectors, prepared especially for this instrument, are operated with bias voltages of $2.5-3.5$ volts. Provision is available for a very thin film to be mounted over the detectors to protect them from contamination, or, if necessary, to cut down the light entering the detectors (these detectors are quite photosensitive).

\section{The Proton Mode of the Instrument}

The proton mode of the instrument was designed to measure the number and energy of protons from $(\alpha, p)$ reactions in certain elements. The number of protons is usually smaller than the number of scattered alpha particles, both because of the smaller number of elements producing protons, the usually intrinsically lower yield of $(\alpha, p)$ reactions, and because the elements producing protons are frequently present in low abundance. The total area of the four proton detectors is, therefore, made as large as possible $\left(-4 \mathrm{~cm}^{2}\right)$, and they are placed closer to the sample than are the alpha detectors. Fortunately, because of the longer range in materials of protons, and because not as much energy or angular resolution is needed in the proton system, such 
a geometrical arrangement is acceptable. Details of construction of the proton detector system are shown in Fig. 4. The average angle at which protons are detected is $145^{\circ}$, but there is a variation of at least $20^{\circ}$ about this value.

In order not to detect scattered alpha particles in the proton mode, enough absorber $\left(21.1 \pm 0.4 \mathrm{mg} / \mathrm{cm}^{2}\right.$ of gold) is placed over the detectors to absorb out even alpha particles with the original energy. (Aluminum is not suitable for this purpose because protons are produced in it by scattered alpha particles.) The thickness of the absorbers must be matched sufficiently to make the four proton detectors have the same energy response to protons. Likewise, variation in the thickness must be minimized. to avoid degradation of the energy resolution of the system.

In view of the low proton rates expected from typical rocks, and the expected cosmic-ray background, the proton detectors are backed up by guard detectors (see Fig. 4) electrically connected in such a way that a particle going through both detectors will not be registered. This anti-coincidence shielding does not affect protons from the sample, since they do not have enough energy to get through the lower detector.

The proton mode of the instrument has been the more difficult to operate. The four detectors are, of course, harder to match in energy response than are the two alpha detectors. In addition, the larger electrical capacity leads to a higher noise level in the first stage of the electronics. Perhaps the most serious operational difficulty is the lack of a monitoring method 
to excite the system through the absorbers. Use of a $\mathrm{Th}^{228}$ standard (having $8.78 \mathrm{MeV}$ alpha particle) has been only partially successful: the energy of this alpha particle after going through the absorber is degraded so much that the peak is broad and is only a qualitative monitor of the behavior of the system.

A final difficulty with the proton system arises from the high voltage needed to produce a sensitive depth in the silicon detector large enough to absorb $6 \mathrm{MeV}$ protons (even after going through the absorber). The detectors used require 70 volts to get a sensitive depth of 200 microns. Changes in leakage current in the electronics can affect the voltage across the detector sufficiently to change the depletion depth and so the appearance of the high-energy part of the proton spectrum. Recent models of this instrument use lithium-drifted detectors, which require much lower bias voltages, and which can be made to be much more stable at the temperature extremes ${ }^{5}$ anticipated on the moon.

\section{ELECTRONICS}

A. Over-all Design

The electronic system associated with the instrument is designed to provide bias voltages and thresholds for the semiconductor detectors, linear amplification for their output pulses, two 128-channel pulse-height analyzers which convert the analogue output of the amplifiers into seven binary digits, three buffer registers for temporary storage of this information, and provision for readout of the register content into the spacecraft transmission system. The electronics also provides means of blocking 
the outputs of the individual detectors by command (from earth) in case of malfunction. In the proton system, the electronics also includes a rejection system for pulses in the detector when they are coincident with a pulse in a guard detector.

These electronics were adapted from circuits originally designed for cosmic-ray studies on satellites. ${ }^{6}$ weight and power considerations and established experience were given over-riding priority in the design. Thus, no local memory (other than the buffer registers) is provided; the instrument is designed for the transmission of the characteristics of each pulse, essentially in real time, down to earth for accumulation and further data analysis on the ground. At the data rates expected ( $\sim$ per second in the alpha mode and 0.1 per second in the proton mode) and with the band widths available for transmission (2200 bits/sec and $550 \mathrm{bits} / \mathrm{sec}$, respectively), this design, incorporating, as it does, some time smoothing of the data, appears adequate for the nominal operating situation on the moon.

\section{B. Block Diagrams}

The electronics of the instrument are partly in the head unit and partly in the electronics package. The portion in the head consists primarily of the linear circuitry. A block diagram of this portion is shown in Fig. 5. This electronics converts a signal output from the semiconductor detectors, after amplification by a charge-sensitive amplifier, into an analogue pulse whose duration is proportional to the energy deposited in the detector (after subtraction of a threshold amount - equivalent 
to about $400 \mathrm{KeV}$ ). The height-to-time converter is of the Hutchinson-Scarrott type. This analogue output is transmitted. over an - $10 \mathrm{ft}$. cable to the electronics package in the spacecraft. A $6-\mathrm{MeV}$ event in a detector produces a pulse around one milli sec long.

At the Mixer circuit in the head, the outputs of the two alpha detectors are combined before further common processing. At this point, the output of either detector can be blocked by command from earth in case of malfunction.

A separate, parallel, electronics system handles the proton detector outputs (proton mode). The outputs of the four proton detectors are combined at the Mixer stage. Associated with each proton detector is a guard detector. The output from any one of these guard detectors, if greater than a threshold, blocks the linear gate for $3 \mathrm{\mu sec}$, while any associated signal from a proton detector is delayed for $-2 \mu \mathrm{sec}$ via a delay line. This prevents the processing of events coincident with pulses from the guard detectors. In the proton mode, the separate switches block the outputs of both a particular detector and its guard.

In addition to this electronics, the head contains a primitive temperature control system. This consists, firstly, of a temperature sensor which provides a temperature reading of the electronics-detector complex and transmits this information to earth. In addition, there is a 5-watt heater that can be turned. on either in a programmed manner, or by earth command, if the temperature falls below a pre-arranged level $\left(\sim \mathrm{O}^{\circ} \mathrm{C}\right)$. 
The electronics package, on the spacecraft, receives the analogue signal from the head, converts it into digital form, provides a small amount of data smoothing, and then routes the digitized information about a given event to the spacecraft telemetry system for transmission down to earth. Again, there are completely parallel systems for both alpha and proton modes of the instruments. In addition, the electronics package contains the power supplies and the control logic for the instrument. A block diagram of the components of the electronics package is shown in Fig. 6 .

The output of the instrument in both alpha and proton modes is a nine-bit word, of which seven bits identify which of 128 possible channels represent the energy of the registered event. Two extra bits are added, one before transmission to identify the start of the word and one at the end of each word, as a parity check on transmission errors. The transmission rates are 2200 bps for the alpha mode and 550 bps for the proton mode. The energy scale of the instrument is about $55 \mathrm{KeV}$ per channel, with a threshold of about $400 \mathrm{KeV}$. Pulses corresponding to events with energy greater than the range of the analyzers are routed to channel 126 (overflow channel).

\section{PERFORMANCE OF THE INSTRUMENT}

This prototype instrument has now been in operation for more than two years. Except for two early detector failures, the performance has been reliable. During the period of active use during the first nine months after completion - there were minor drifts in the gain and offset of the alpha system. The changes could be monitored using a monochromatic source of alpha particles. No changes were observed in the proton system during this period. 
An instrument designed to operate on the lunar surface should be capable of satisfactory performance under a wide range of temperature conditions. The first prototype has been operated at temperatures from $-40^{\circ} \mathrm{C}$ to $+50^{\circ} \mathrm{C}$. The over-all gain changes in the detector-electronics system were somewhat larger than desirabie ( $\sim$ 8\% in the alpha mode and $\sim 5 \%$ in the proton mode) over this temperature range. There were also up to $2 \%$ changes in response at $\sim 6 \mathrm{Mev}$, depending on the number of detectors operating.

A second prototype of this instrument has passed many of the special shock, vibration, and temperature tests required of an experiment designed for a lunar mission. This second instrument has taken measurements at both low $\left(-35^{\circ} \mathrm{C}\right)$ and high $\left(+45^{\circ} \mathrm{C}\right)$ temperatures.

The data rates expected with an instrument of this type are so low that background events and electrical noise must be kept as low as possible. The relatively high thresholds in the electronic system lead to background rates (in the absence of radioactive contamination) of less than 0.5 events $\mathrm{min}^{-1}$ in the alpha mode and less than 2 events $\mathrm{min}^{-1}$ in the proton mode.

On the other hand, the presence of a small ( $\leq 1$ event/min) contamination close to the detector can be a convenient way of monitoring the behavior of the instrument during long measurements. A suitable nuclide for this purpose is $\mathrm{Es}^{254}$. Its alpha energy $(6.42 \mathrm{MeV})$ is sufficiently high not to interfere with the spectra of alpha particles or protons produced by samples. 
The first prototype has been used to establish the response of this type of instrument to a whole range of elements, as well as the capability to analyze complex rocks. The results of this study are presented elsewhere. 7 The techniques of measurement and examples of elemental response curves are given in this report.

The instrument can be checked out in air by use of a source block that presents alpha radioactive sources close to each detector. Although the resolution of the instrument, when checked in this way, is poor due to variable air absorption, this is a convenient way to establish the electronic capability of the instrument.

After the high-level sources are inserted into the instrument, it can be checked in air. The response under these conditions is illustrated in Fig. 7. The alpha mode, even in the poorly defined geometry of a gaseous sample, clearly identifies the nitrogen and oxygen in the air. The level of response, as well as some qualitative features of the spectra, depends on the pressure of air in the instrument. There are negligible events in the proton mode when the instrument is operated at one atmosphere of air, because of the unfavorable geometry for the $N(\alpha, p)$ products getting into the proton detectors.

Because of the short range of 6.11 alpha particles, quantitative results on samples must be obtained in vacuum. This, of course, will be no problem on the lunar surface. In the present studies, the head was kept in a vacuum chamber $(\mathrm{P}<1 \mu \mathrm{Hg})$, with 
samples and test sources being presented through a vacuum lock. After insertion of the sample, the head could be lowered to get a reproducible - or known - distance between the source and detectors and the sample.

Figure 8 illustrates the response of the instrument to the set of monochromatic alpha particles present in a source of $\mathrm{Th}^{228}$ in equilibrium with its daughters. This source was prepared in an isotope separator, with an accelerating potential of $40 \mathrm{KeV}$. Figure 8 shows that the over-all resolution of the instrument in the alpha mode is less than 1.8 per cent (full width at half maximum) in the region $5-7 \mathrm{MeV}$.

Using data of the type of Fig. 8, the energy scale of the instrument can be established. For the first prototype instrument, on a reference date, the relation between channel number, $N$, and energy, $E$ (in $\mathrm{MeV}$ ), was established to be (for the alpha mode):

$$
N=16.16 \mathrm{E}-4.31
$$

For the proton mode, less direct evidence (because of the gold foil over the absorbers) gave:

$$
\mathrm{N}=18.0 \mathrm{E}-11.4 \text {. }
$$

Precision electronic pulser calibration of a second prototype has shown that the pulse-height analyzers of the type used in these instruments is linear to better than a few per cent down to about channel 10 . 
Figures 9 and 10 show the response of the instrument to several pure elements in the alpha and proton modes, respectively. The data are from several combined runs, with the information normalized to a source strength of $6.7 \times 10^{10} \mathrm{~d} / \mathrm{m}$, and a measurement time of $1000 \mathrm{~min}$. The data has been corrected for small electronic shifts of the instrument, and the background has already been subtracted. The data of Figs. 9 and 10 are similar to those obtained in the report of Patterson, Turkevich, and Franzgrote. ${ }^{2}$ Together with the response from other elements, these represent a "library" on the basis of which the response from a complex substance can be used to determine the chemical composition of the substance.

\section{ACKNOWLEDGMENTS}

The development and construction of this instrument has involved the collaboration of the staff of the Laboratory for Astrophysics and Space Research under the general supervision of James E. Lamport. Robert Takaki was responsible for designing many of the electronic circuits, Myron Weber and Ed Blume for much of the mechanical design, and Anthony J. Tuzzolino and Murray Perkins for the fabrication of many special detectors needed in this work. At the Argonne National Laboratory, Jerome Lerner prepared the $\mathrm{Th}^{228}$ source and Dale Henderson performed many measurements on the $\mathrm{Cm}^{242}$ sources used in this work. At the Jet Propulsion Laboratory, Glenn Sisk and Timothy Harrington contributed, particularly to the adaptation of the instrument to Surveyor requirements. 
This instrument was developed and tested under partial support from the National Aeronautics and Space Administration at the University of Chicago (under grant NsG-127-61 and subcontracts JPL-NASA 950750 and 950315 with the Jet Propulsion Laboratory). The work at the Argonne National Laboratory was supported by the United States Atomic Energy Commission; that at the Jet Propulsion Laboratory by contract NAS 7-100 of the National Aeronautics and Space Administration. 


\section{REFERENCES}

*

Work at the University of Chicago supported in part by the National Aeronautics and space Administration under grant NSG-127-61 and subcontracts JPI-NASA 950750 and 950315 with the Jet Propulsion Laboratory, at the Argonne National Laboratory by the United States Atomic Energy Commission, and at the Jet Propulsion Laboratory by contract NAS 7-100 of the National Aeronautics and Space Administration.

† Present address: Tulpenhofstrasse 29, Offenbach Am Main, Germany •

* Present address: University of Michigan, Box 618 Ann Arbor, Michigan.

1 A. Turkevich, Science 134, 672 (1961).

2 J.H. Patterson, A. Turkevich, and E. Franzgrote, J. Geophys. Res. 70, 1311 (1965) .

3 E. P. Horwitz, C. A. Bloomquist, H. W. Harvey, D. Cohen, and I. J. Basile, "The Purification of 10 Curies of $\mathrm{Cm}^{242}$, "ANL-6998 (1965)

4 N. A. Eskind and H. Mark, J. Geophys. Res. 67, 4867 (1962).

5 A. J. Tuzzolino, J. Kristoff, and M. A. Perkins, Nucl. Instr. Meth. 36, 73 (1965); Nucl. Instr. Meth. 37, 204 (1965).

6 see e.g., C. Y. Fan, G. Gloeckler, and J. Simpson, J. Geophys. Res. 70, 3515 (1965); S. M. Comstock, C. Y. Fan, and J. Simpson, Astrophys. J. (1966)(to be published).

7 A. Turkevich, K. Knolle, E. Franzgrote, and J. H. Patterson (in preparation). 
FIG. I

Alpha-Scattering Instrument for Surface Analysis. The two units of the instrument are the boxes on the table on the right side of the figure. The deployable head is the unit on the right. The electronic racks include spacecraft data handling simulators and test and monitor equipment. 


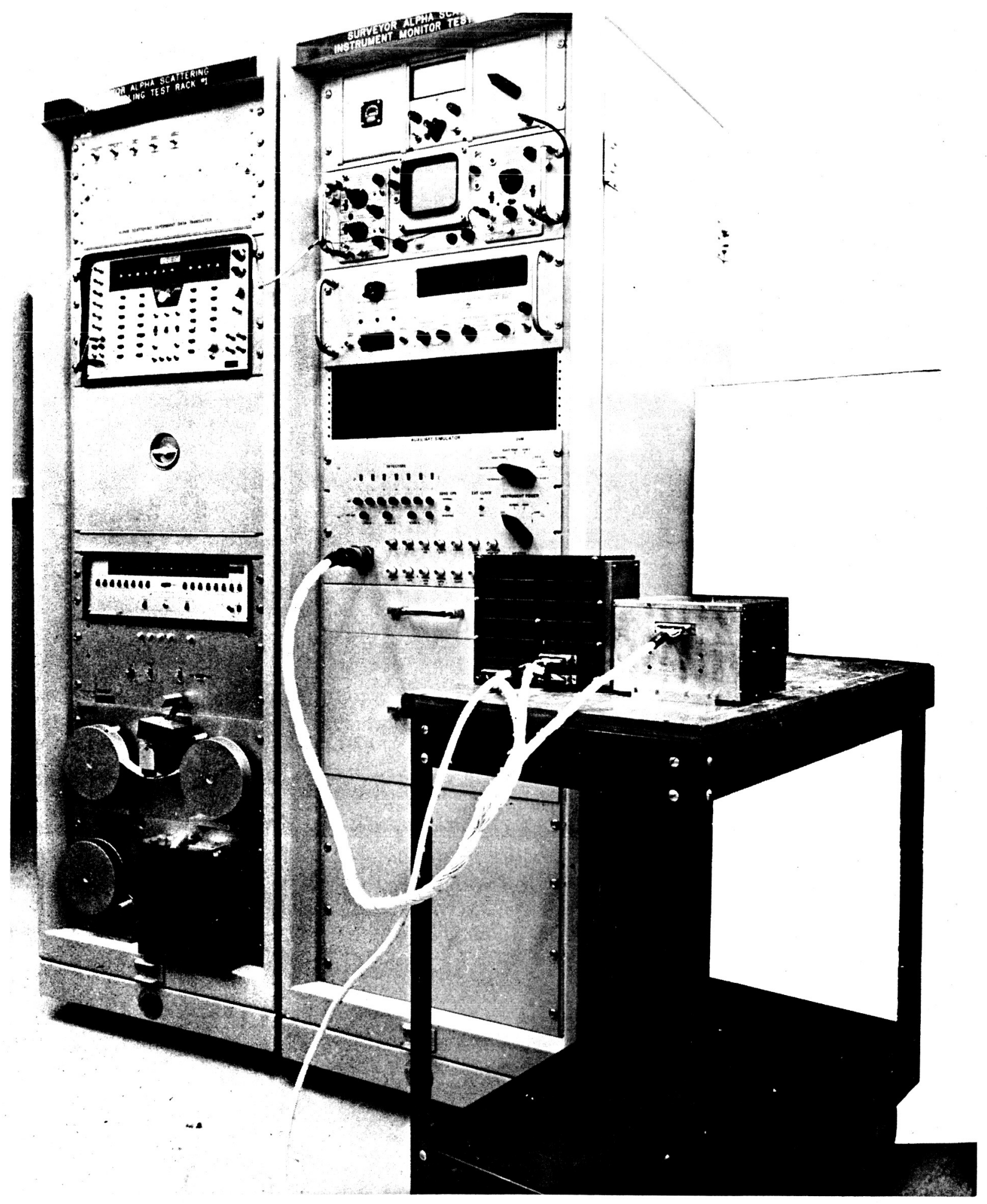

Fig.I 
FIG. 2

Alpha-Scattering Instrument: View up through bottom opening of Head Unit. The six collimated alpha sources, the two alpha detectors, and the four proton detectors are identified. The alpha sources and detectors are $-5.7 \mathrm{~cm}$ up from the opening; the proton detectors are about $5 \mathrm{~cm}$ away (see Fig. 3) 


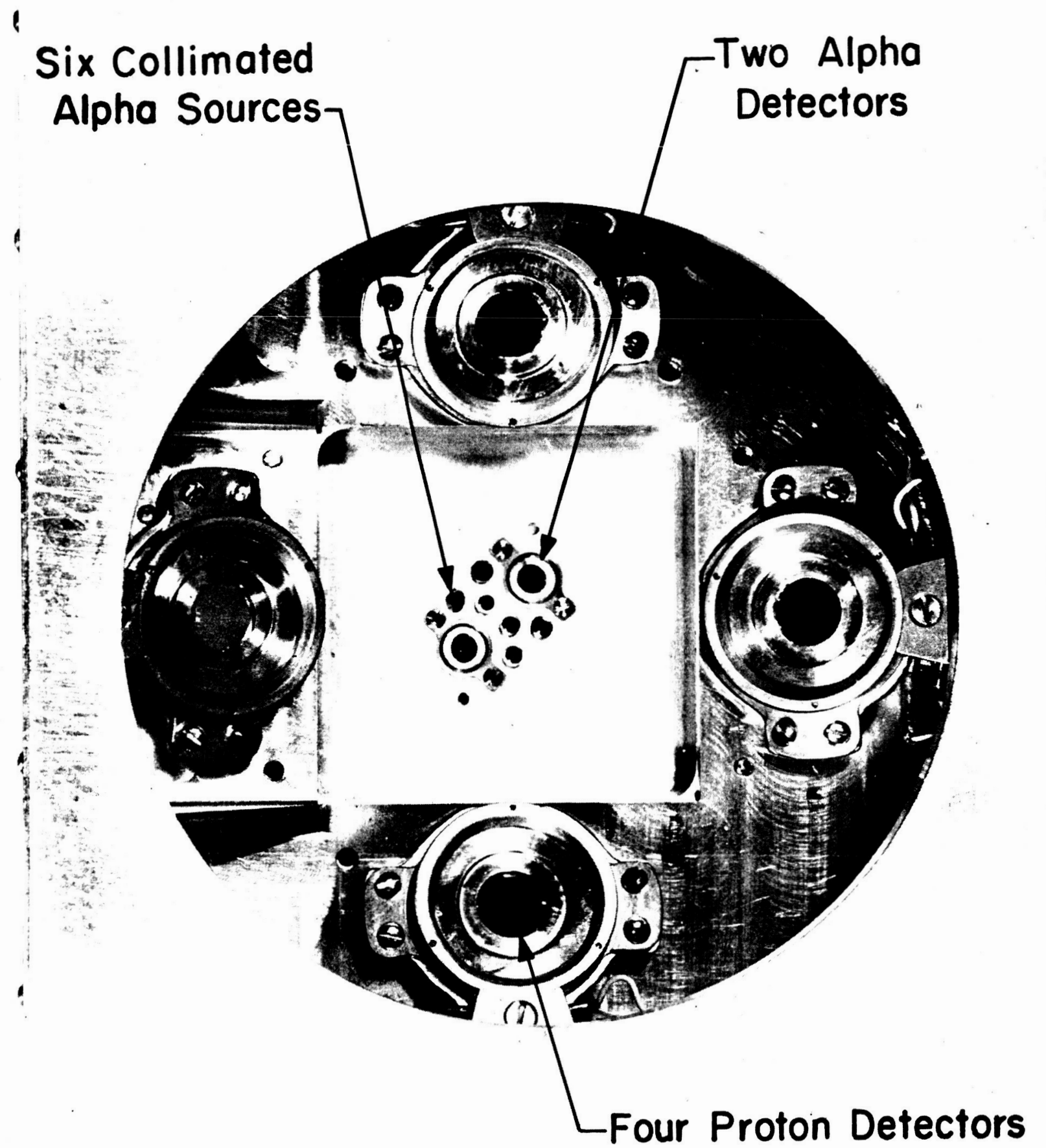

Fig. 2 
$\underline{F I G \cdot 3}$

Alpha-Scattering Instrument: Some geometrical relationships between parts of the head unit. The top part of the figure shows a horizontal cross section about $2 / 3$ of the way up the head unit. The bottom part of the figure shows a vertical cross section approximately through the center of the unit. The six source holders ( $s)$, the two alpha detectors $(\alpha)$, and the four proton detectors $(p)$ are shown, together with typical paths for the alpha particles and protons. 

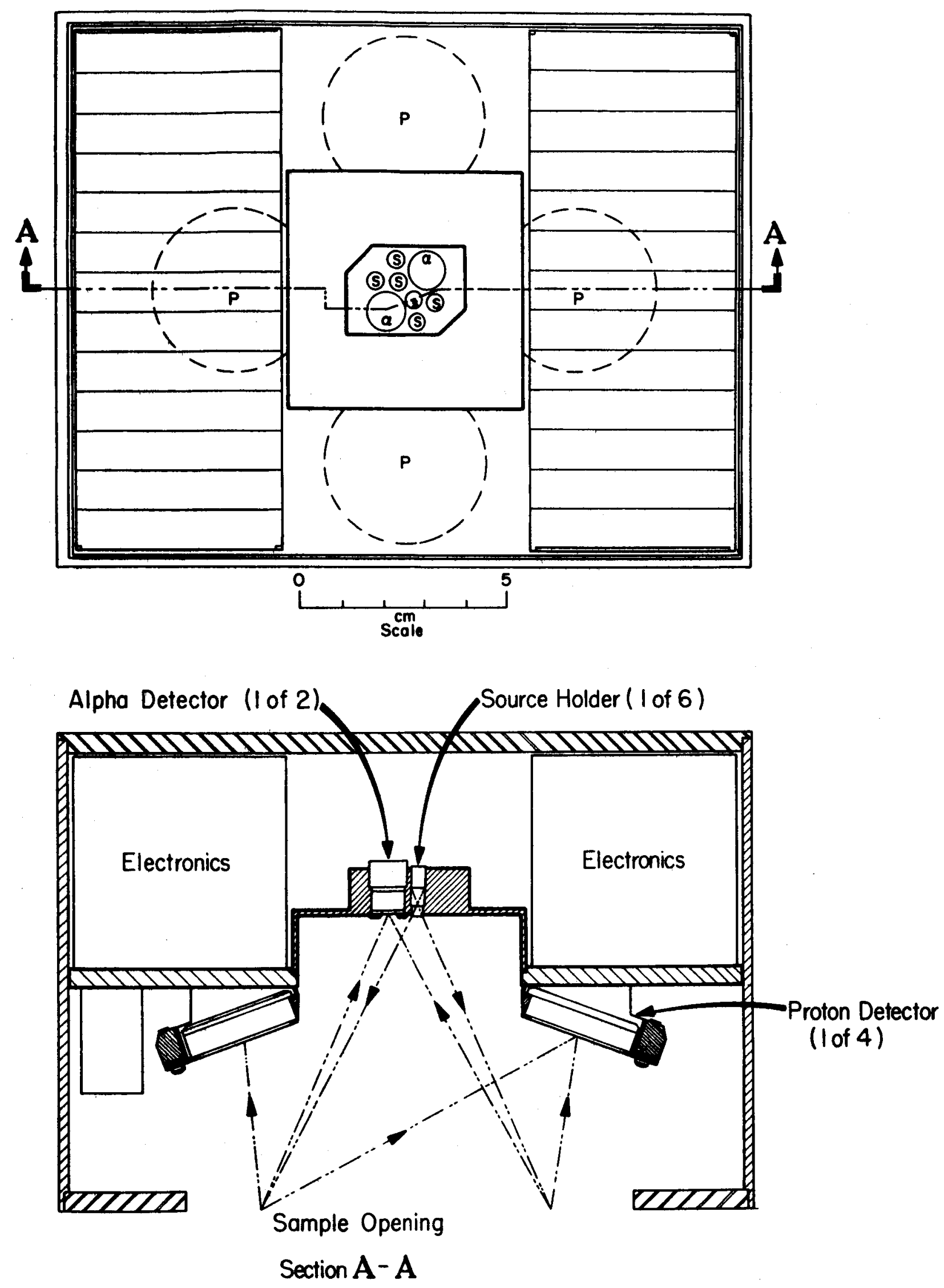

Fig. 3 
FIG. 4

Alpha-Scattering Instrument. Details of construction of Alpha Source Holders, Alpha Detectors, and Proton Detectors. 


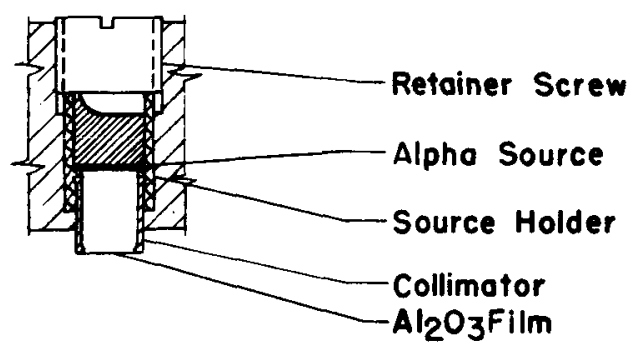

SOURCE ASSEMBLY

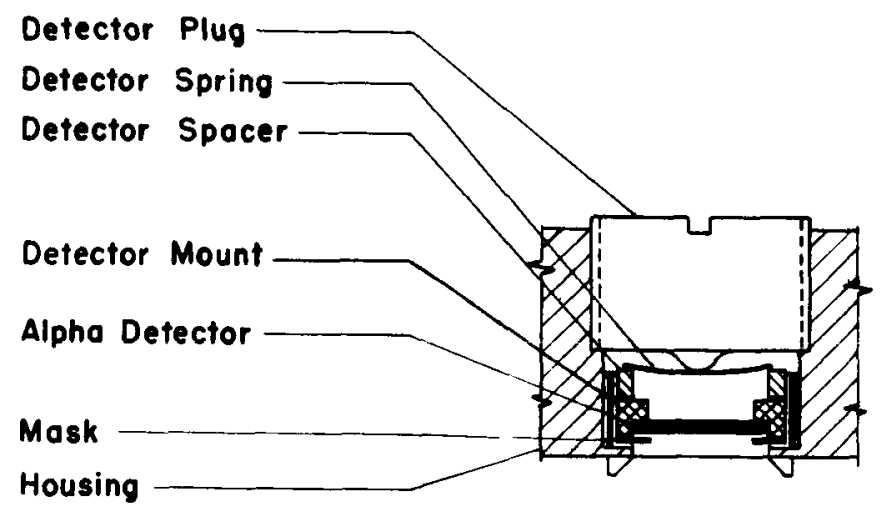

ALPHA DETECTOR ASSEMBLY

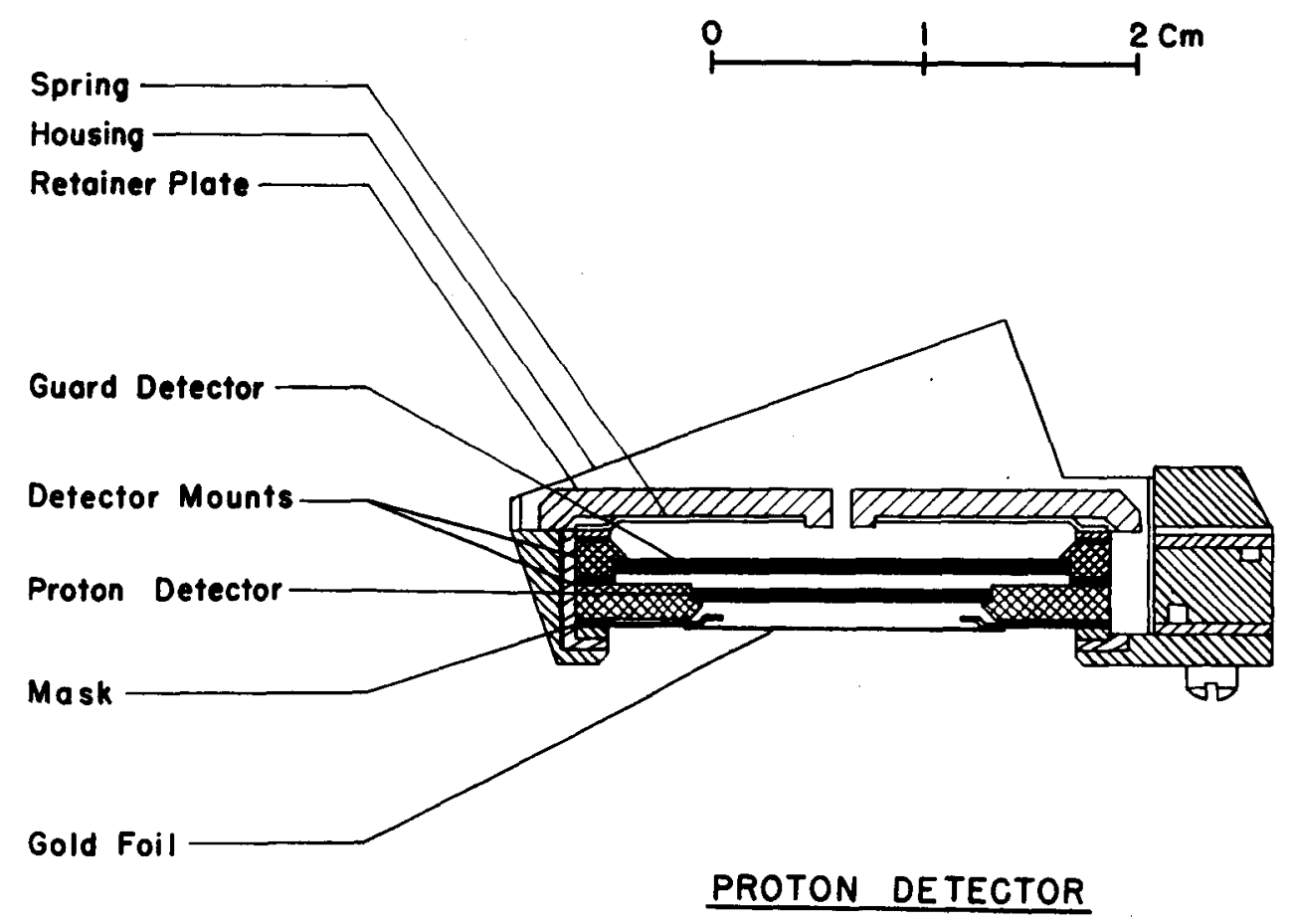

Fig. 4 
FIG. 5

Alpha-Scattering Instrument. Block Diagram of Electronics in Head Unit.

Legend:

$$
\begin{aligned}
& \alpha-1, \alpha-2: \text { Two silicon semiconductor detectors } \\
& \text { arranged to detect alpha particles. } \\
& \text { P-I, ... P-4: Four silicon semiconductor detectors } \\
& \text { arranged to detect protons. } \\
& \text { G-1, ... G-4: Four guard detectors in anticoincidence } \\
& \text { with the proton detectors to lower the } \\
& \text { background in the proton detectors. } \\
& \text { B: Appropriate bias voltages for the silicon } \\
& \text { detectors. } \\
& \text { S: Electronic switches, actuated by ground } \\
& \text { command, that can block outputs of } \\
& \text { separate alpha detectors or separate } \\
& \text { proton-guard detector combinations. }
\end{aligned}
$$




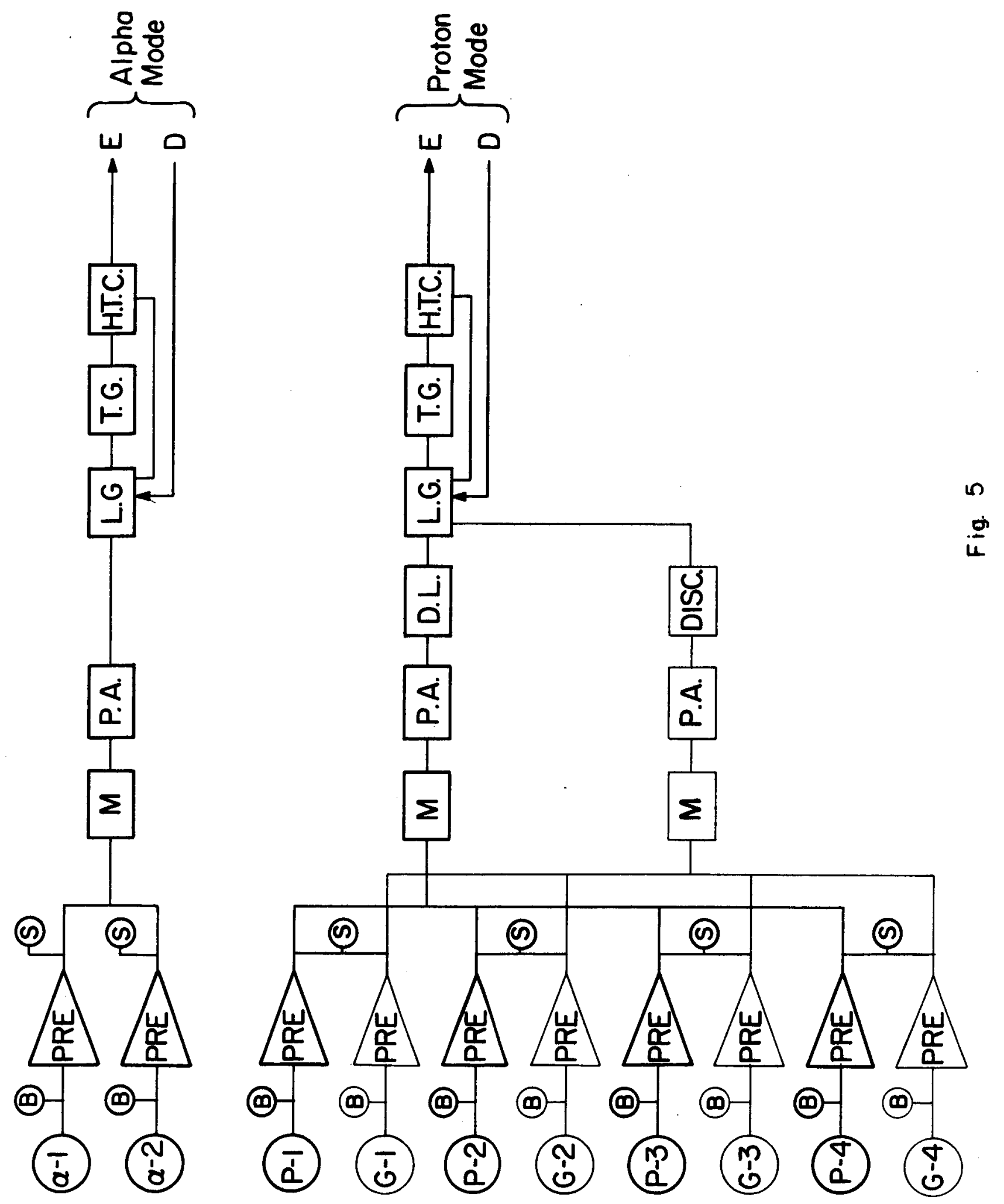


FIG. 6

Alpha-Scattering Instrument. Block Diagram of Electronics in Electronics Unit.

Legend :

E: Analogue signal from Head Unit.

D: Signal to Head. Unit to block processing of new pulses when buffer counters are filled.

DLC: Delay Line Clock.

C : Seven-bit Counter.

BS: Two buffer storage counters for time smoothing of data.

PSC: Parallel to serial converter.

PG: Parity Generator.

G: Signal for synchronization of data output with spacecraft clock.

F: Data output to spacecraft ( $\mathrm{s} / \mathrm{c}$ ).

P: Power supply converting 29 volt spacecraft voltage to loov, 29V, and 7-volt levels needed in instrument electronics.

M: Electronic memory for processing commands to turn outputs of individual detectors on and. off. 

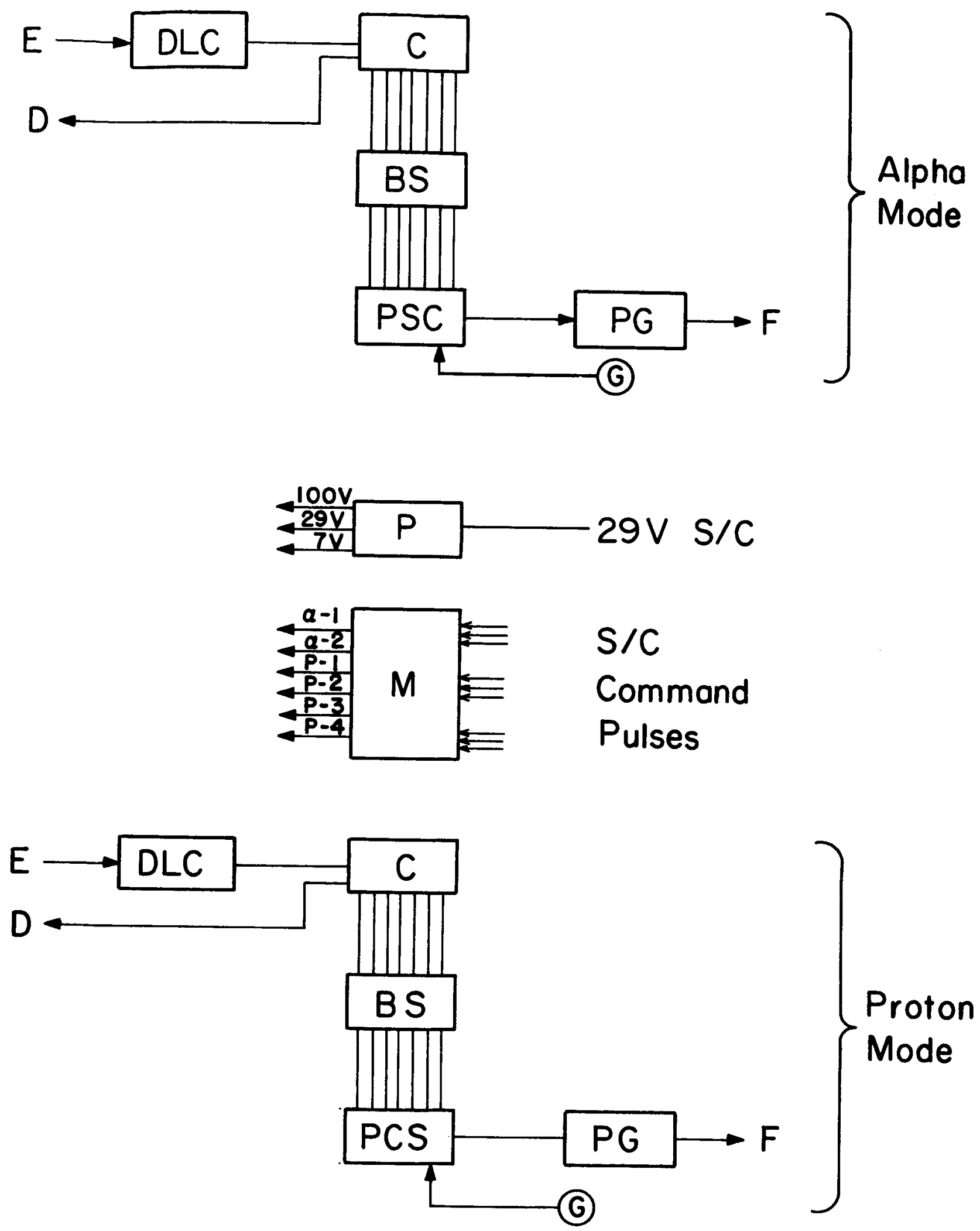

Fig. 6 
FIG. 7

Spectrum Obtained in Alpha Mode of Alpha-Scattering Instrument, $\mathrm{P}-1$, with air in the instrument. The ordinates are events per channel in a 75 min. measurement with a total source strength of $7 \times 10^{10} \mathrm{~d} / \mathrm{m}$; the abscissae are channel numbers of the pulse-height analyzer of the instrument. The relation between channel number and energy is indicated in the text. The energy (channel) regions in which the principal constituents of air contribute are indicated at the top. 


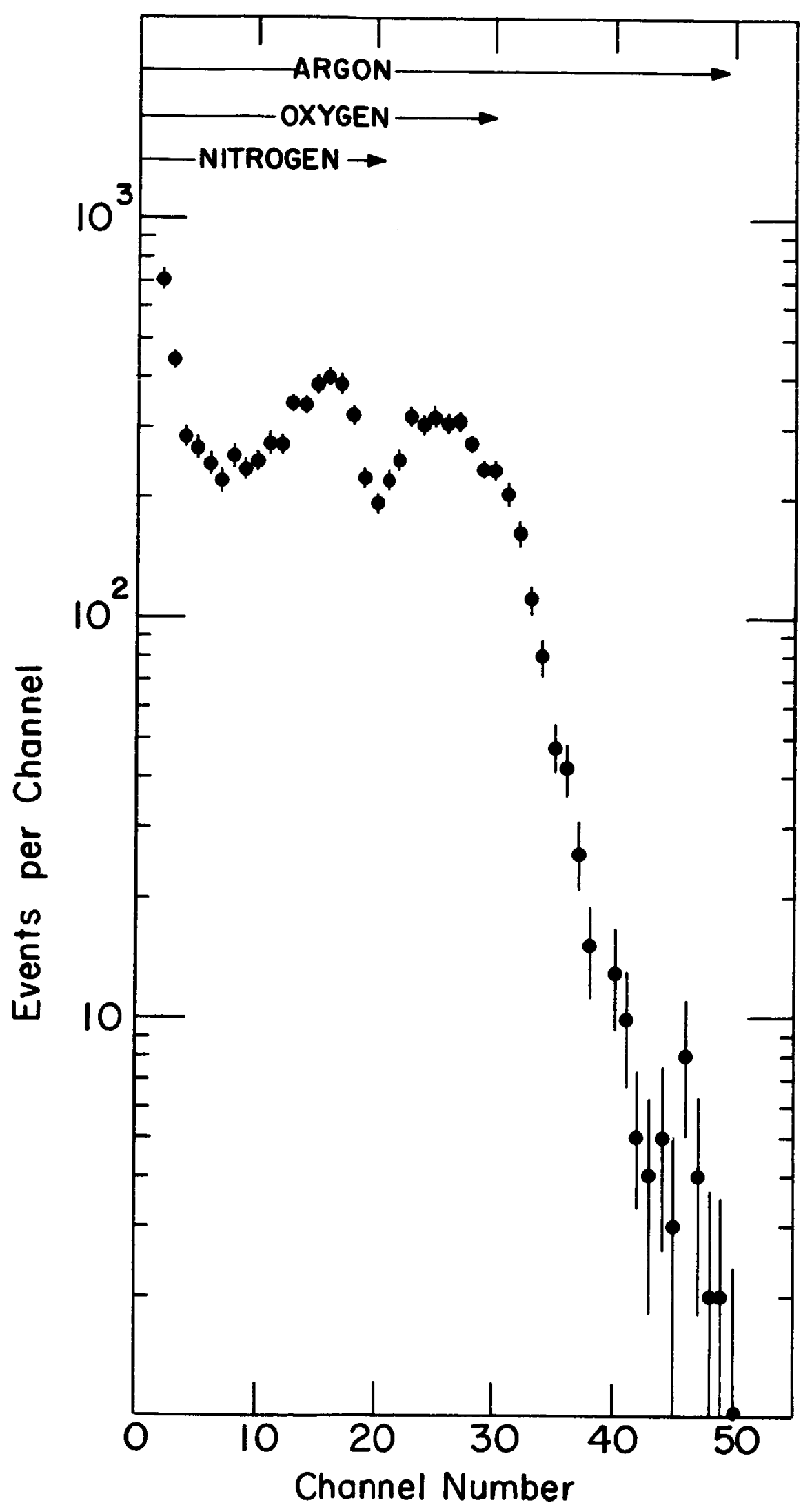

Fig. 7 
FIG. 8

Response of Alpha-Scattering Instrument, P-I (Alpha Mode) to $\mathrm{Th}^{228}$ (in equilibrium with its daughters). The literature values of the energy of the Alpha-Particle Groups are indicated. at the top of the figure. The highest energy alpha group in this series ( $8.78 \mathrm{MeV}$ ) has too high an energy for the pulseheight analyzer of the instrument and appears in the overflow channel (126). 


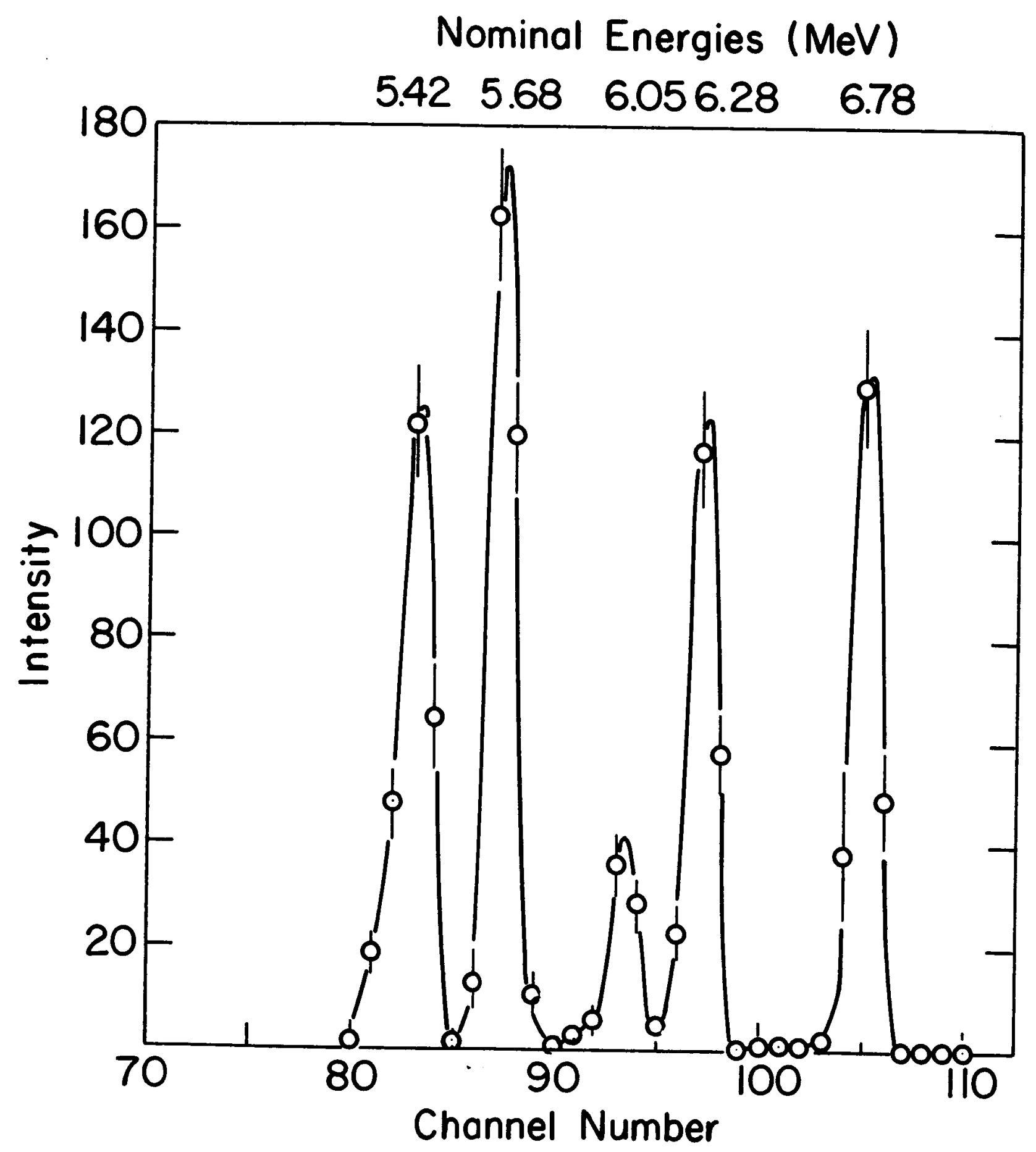

Fig. 8 
FIG. 9

Response of the Alpha-Scattering Instrument in the Alpha Mode to Three Pure Elements. The data are for carbon, magnesium, and iron. The ordinates are events per channel registered in 1000 min., as a function of channel number. The source strength was $6.7 \times 10^{10} \mathrm{~d} / \mathrm{m}$. 


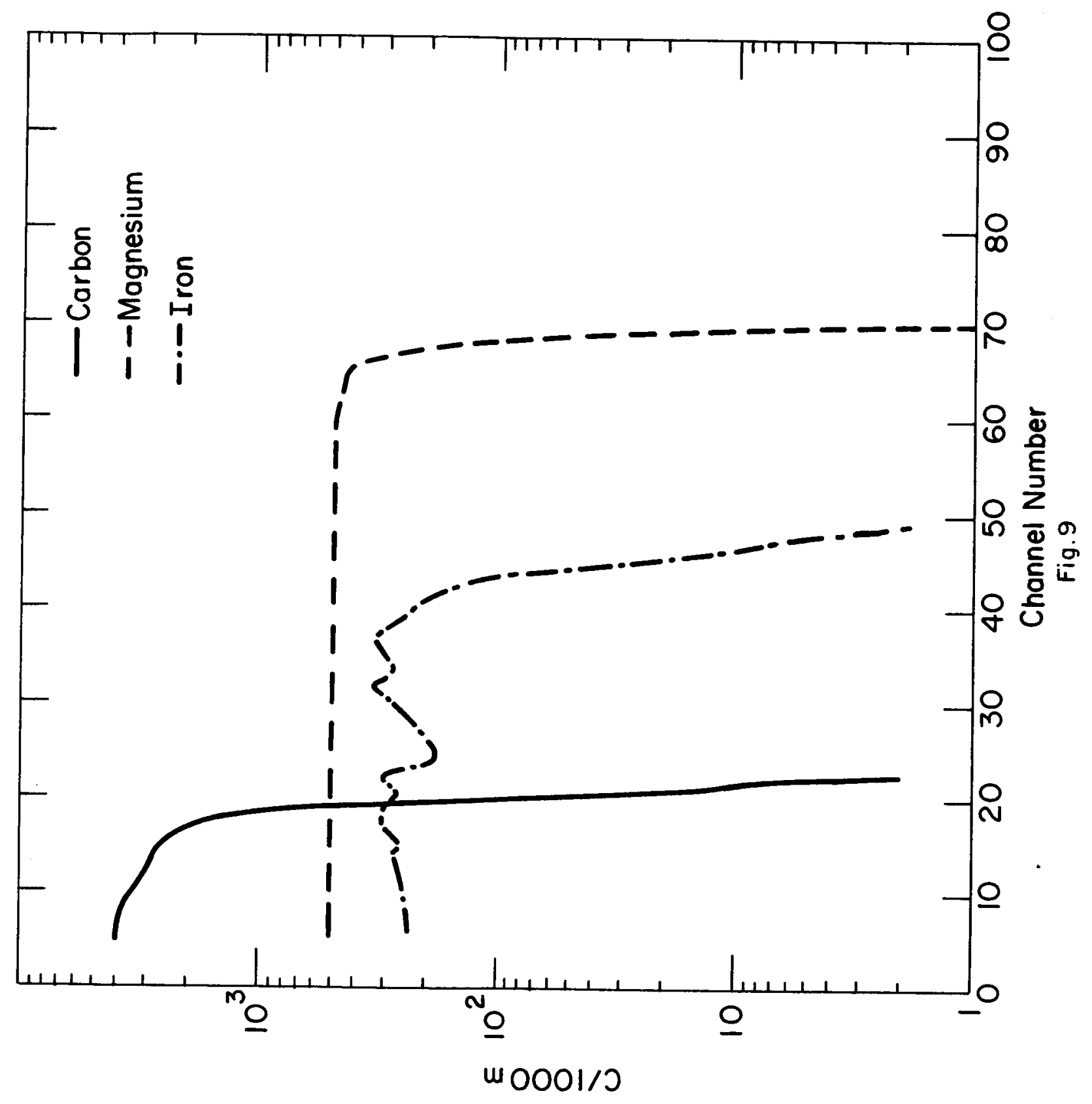




\section{FIG. 10}

\section{Response of the Alpha-Scattering Instrument in Proton}

Mode to Three Pure Elements. The data are for aluminum, silicon, and phosphorous. The ordinates are events per channel registered in $1000 \mathrm{~min}$., as a function of channel number. The source strength was $6.7 \times 10^{10} \mathrm{~d} / \mathrm{m}$. 
$\because$

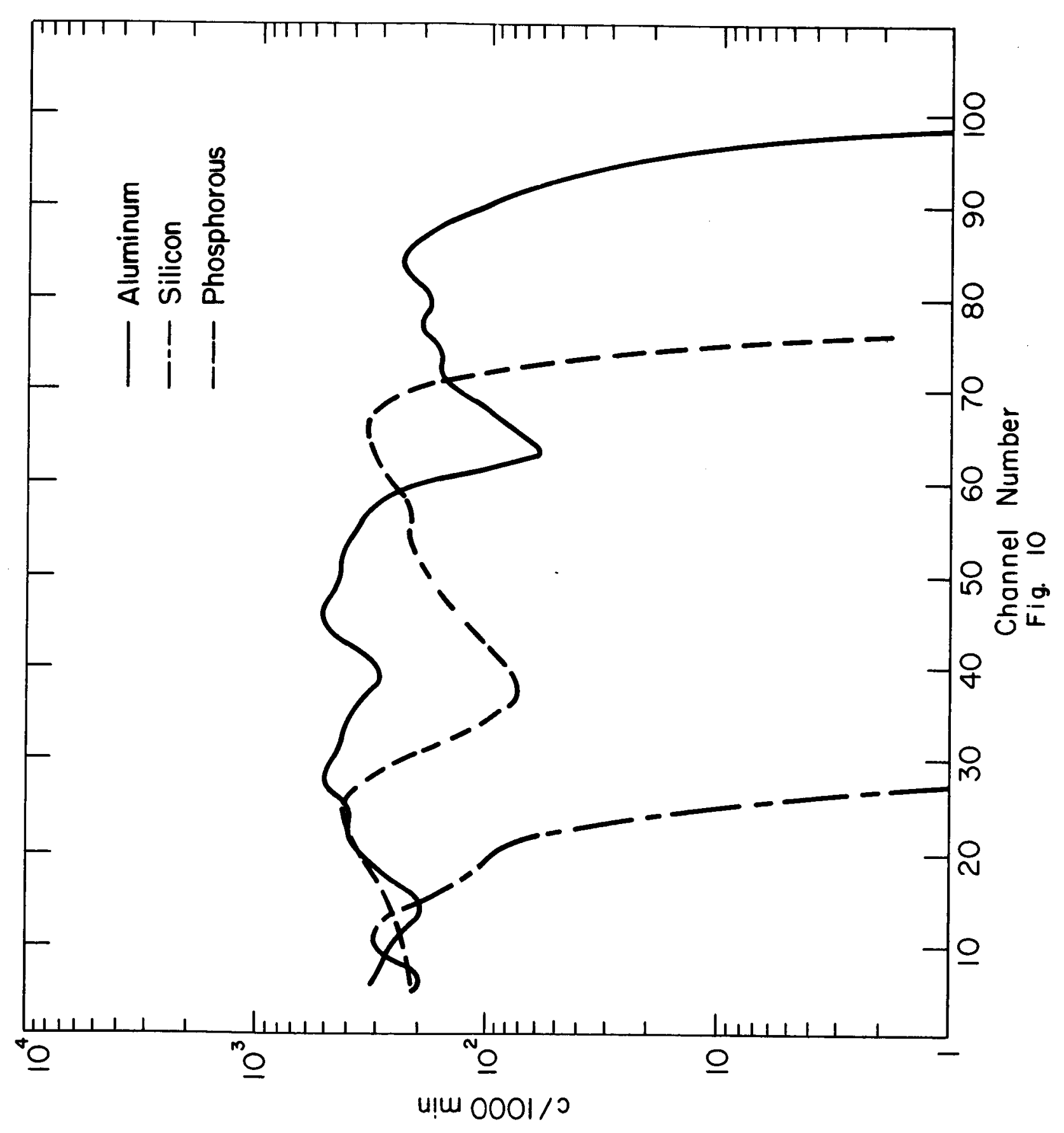

